\title{
Hollow fibers made from a poly(3-hydroxybutyrate)/poly-e-caprolactone blend
}

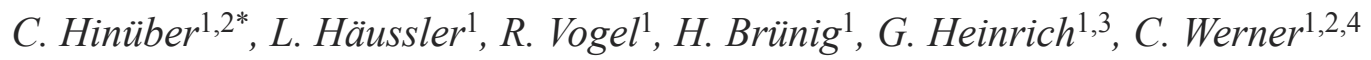 \\ ${ }^{1}$ Leibniz-Institut für Polymerforschung Dresden e.V., Hohe Str. 6, D-01069 Dresden, Germany \\ ${ }^{2}$ Max Bergmann Zentrum für Biomaterialien Dresden, Budapester Str. 27, D-01069 Dresden, Germany \\ ${ }^{3}$ Technische Universität Dresden, Institut für Werkstoffwissenschaft, Helmholtzstrasse 7, D-01069 Dresden, Germany \\ ${ }^{4}$ Technische Universität Dresden, Zentrum für Regenerative Therapien Dresden, Tatzberg 47, D-01187 Dresden, Germany
}

Received 2 November 2010; accepted in revised form 20 January 2011

\begin{abstract}
Since poly(3-hydroxybutyrate) (PHB) is inherently brittle and possesses poor elastic properties, hollow fibers produced by melt spinning from pure PHB, as described in our earlier study [Macromolecular Materials and Engineering, 2010, 295/6, 585-594], do not meet the required needs regarding the mechanical performance. Besides hardly available PHB copolymers, also blend systems are known to enhance material properties and have thus been considered to be eligible to fabricate flexible or rather pliable hollow fibers based on PHB. Blends of PHB and poly- $\varepsilon$-caprolactone (PCL) are promising for the application in tissue engineering due to the inherent biocompatibility and biodegradability. A wide range of PHB/PCL compositions have been prepared by melt extrusion. Thermal and mechanical properties of the obtained specimens were analyzed in order to identify miscibility and degree of dispersion as well as to determine the influence on the overall mechanical performance. Even though these constituents are known to be immiscible, PHB/PCL 70/30 was proven to be an adequate composition. This blend showed a highly increased elongation and was found to be easily processable by melt spinning compared to pure PHB. From this blend well defined dimensionally stable bendable hollow fibers were fabricated.
\end{abstract}

Keywords: biodegradable polymers, mechanical properties, PHB, PCL, melt spinning

\section{Introduction}

Poly(3-hydroxybutyrate) (PHB), produced by microorganisms in order to store carbon as energy resource, is a promising material for applications requiring biocompatibility, non-toxicity as well as biodegradability $[1,2]$. Therefore, investigating, developing and modifying PHB based materials are of great interest in regenerative medicine. In earlier studies it was shown that PHB can be fabricated to multifilament fibers with textile properties using a highspeed melt spinning and drawing technology and further processed into textile scaffolds and wound healing patches [3-6]. Recently, we demonstrated that dimensionally stable hollow fibers from PHB can be spun [7].

For instance, latest concepts in neuro-tissue engineering call for biocompatible, biodegradable and biofunctionalized polymers as temporary nerve guidance conduits. The strategies in neurosurgery tend to treat nerve injuries by means of tubulization techniques to initiate and stimulate nerve regeneration as explained in detail by Battiston et al. [8], and Ruiter et al. [9]. According to these authors, an ideal nerve guidance conduit performs better than an autograft, offering at least following features: biocompatibility; a biodegradable, non toxic mate-

\footnotetext{
${ }^{*}$ Corresponding author, e-mail: hinueber@ipfdd.de

(c) BME-PT
} 
rial; certain flexibility and certain porosity that guarantees permeation of nutrients and oxygen but avoids ingrowth of competitive cells. Besides this, an internal framework or a multichannel structure is regarded beneficial $[9,10]$. Furthermore, a defined biomolecular functionalization including appropriate growth factors and/ or cells (e.g. Schwann cells) seems to be essential $[11,12]$. Various degradable materials are under investigation: e. g. Matrigel, Collagen, PLLA, PLGA, PCL $[9,13,14]$. However, tubular structures made of very soft or fast degrading materials might collapse or kink before regeneration has been completed and will strongly constrict or even obstruct tissue regeneration [15]. Since PHB is a stiff and rather slowly degrading biopolymer, it has received more attention in neuronal regeneration research $[11,16,17]$. In contrary to time-consuming manual methods providing three dimensional structures via solvent casting methods, dip coating, wrapping of sheets or braiding of fibers around canules, the thermoplastical processability of PHB allows for a fast and continuous production of tube-like structures via melt spinning, which is the most efficient process to produce fibers [7]. Despite of brittleness and thermooxidative degradation, the feasibility of spinning dimensionally stable hollow fibers from PHB was shown for the first time. However, the main drawback of this material, poor mechanical performance due to its inherent rigidity, still remains an issue. Therefore, the following study addresses the modification of PHB to significantly enhance ductility. Besides PHB-copolymers exhibiting good elastic properties, blending with certain biopolymers seems to be a reasonable option. Blending is a known physical method to vary and tailor material properties by mixing two homopolymer components either in molten or in dissolved state. The aim of blending is to achieve intermediate or even superior properties while preserving the major characteristics of the pure components.

PHB has been blended with several other biocompatible and biodegradable polymers, for instance poly(ethylene oxide), poly (L-lactide), poly (L-lactic acid) or poly-e-caprolactone (PCL), in order to improve weak properties [18, 19]. PCL in particular, a synthetic semicrystalline polyester also belonging to the category of biocompatible and biodegradable polymers, is considered as an interesting blend component due to the high ductility and thermal resistance. In fact, the melting temperature of PCL is significantly lower compared to PHB but PCL does not undergo rapid thermal degradation, hence processing far above melting temperature is not critical. PCL is generally miscible with a wide variety of polymers acting as a plasticizer and leading to a more flexible material by lowering the glass transition temperature $\left(T_{\mathrm{g}}\right)$ and the Young's modulus $(E)$. However, PCL is known to be immiscible with PHB when blended mechanically or in solution and thus considered not to improve mechanical properties in case of PHB [18, 20-23]. Nevertheless, this blend system is highly discussed in literature due to results that are reported on the attempts to improve miscibility leading to increased ductility and also to tailor degradability. Avella et al. reported about physically blended PHB and PCL. No synergetic effects have been found and material performance was not improved for blends with less than $60 \mathrm{wt} \%$ of PCL. Soft PCL droplets within the PHB phase did not lower the rigidity [18]. Contrary to these findings Lovera et al. [19] showed an increased elasticity. Even though the aim of this study was rather the investigation of the degradation behaviour than the improvement of the mechanical properties of these solution cast blends, tensile tests demonstrated that a PHB/PCL 70/30 blend shows elongation values at break of about $50 \%$ whereas pure PHB tolerates a strain of $10 \%$ only, for nondegraded state, respectively. Lovera et al. also tried to improve miscibility of the PHB/PCL blends by using a chemically modified low molecular weight PCL. Blends with chemically modified PCL were proven to be partly miscible and showed a reduced degradation rate which is interesting in terms of tailoring the blend properties. Avella et al. [18] also reported on miscibility when utilizing low molecular weight PCL. This article reports about the formation of hollow fibers by melt spinning using a particular PHB/PCL blend in order to achieve a bendable hollow structure that is applicable in regenerative medical therapies, for instance as nerve guidance conduit.

\section{Experimental part \\ 2.1. Materials}

High molecular weight poly(3-hydroxybutyrate) (Biocycle ${ }^{\circledR} 1000, M_{\mathrm{w}}=349000$ ) was obtained from 
Biocycle (São Paulo/Brazil). High molecular weight poly- $\varepsilon$-caprolactone $\left(\right.$ capa $^{\circledR} 6800$, PCL, $M_{\mathrm{w}}=$ $120000)$ was purchased from Perstorp UK Ltd. (Warrington/ UK).

Since the used spinning set-up does not include an extrusion or mixing process and thermo-oxidative degradation influences PHB properties quickly, carefully mixing of both polymer components in non molten state is of crucial impact. Polymer blends were prepared by

a) simply mixing of granulates of both components,

b) milling and mixing of fine powder, as well as

c) using a solvent and applying a precipitation procedure.

For spinning experiments, granules of both polymers have been milled into fine powder using a Retsch two step rotor-sieve mill with a sieve size of $0.5 \mathrm{~mm}$ (Ultra Centrifugal Mill ZM 200, Retsch, Haan/ Germany). Since PCL is cereous, both polymers were cooled down in liquid nitrogen for 510 minutes before milling. Thus, the milling procedure was simplified by polymer embrittlement and smudging of the sieve was avoided. Beneficial to the preparation of a homogenous mixture both polymer components were purified and mixed by dissolution in chloroform at $40^{\circ} \mathrm{C}$ and precipitated in methanol. Precipitated polymer mixture was finally separated from solvents by filtration and vacuum dried $\left(115^{\circ} \mathrm{C}, 24 \mathrm{~h}\right)$. Remaining polymer 'flakes' were compacted into $2.5 \mathrm{~cm}$ discs using a hydraulic press and cut into smaller pieces to allow for easy filling.

\subsection{Methods}

\subsubsection{Sample preparation}

For thermal analysis (DSC), visualization (SEM) as well as for mechanical characterization (tensile test) samples of PHB/PCL with varying compositions were compounded from granulates $(\mathrm{G})$ using a microscale twin screw extruder (Micro 5, DSM Research, Delft/Netherlands): PHB/PCL G 100/0, G 90/10, G 80/20, G 70/30, G 60/40, G 50/50. Sample mass was about $3 \mathrm{~g}$ per run. Compounding parameters were set to: $185^{\circ} \mathrm{C}, 80 \mathrm{rpm}$ and $2 \mathrm{~min}$. Besides simple extrusion of 'worm-like' structures, fiber-like structures were extruded by using self-constructed nozzles. No drawing process was applied to these fibers as usually practiced for melt spinning fibers.
Extruded fibers varied in diameter from 0.4 to $1.0 \mathrm{~mm}$. For the purpose of comparison, also a sample from precipitated material (F 70/30) was compounded and prepared for DSC measurements.

\subsubsection{Differential Scanning Calorimetry (DSC)}

The thermal analysis was carried out using differential scanning calorimetry (Q1000, TA Instruments, Dallas/Texas/ USA) in inert nitrogen atmosphere. Calibration was performed with an indium standard. A fresh sample of the compounded material $(\sim 5 \mathrm{mg})$ was prepared for each DSC run. Pieces of adequate size were cut from the extruded strand. Data for a cycle of heating-cooling-heating were collected. All measurements were replicated to verify the collected data. Specimens were exposed to the following DSC regime:

First heating, cooling and second heating scans were performed in a temperature range from $-60^{\circ} \mathrm{C}$ (kept for $5 \mathrm{~min}$ ) to $+185^{\circ} \mathrm{C}$ (kept for $2 \mathrm{~min}$ ) with a heating/cooling rate of $20 \mathrm{~K} \cdot \mathrm{min}^{-1}$. The glass temperature $\left(T_{\mathrm{g}}\right)$, the peak temperatures of melting $\left(T_{\mathrm{m}}\right)$ and crystallization $\left(T_{\mathrm{c}}\right)$ and the onset temperature of crystallization $\left(T_{\mathrm{o}}\right)$ as well as the melting and crystallization enthalpy $\left(\Delta H_{\mathrm{m}}, \Delta H_{\mathrm{c}}\right)$ were determined.

\subsubsection{Tensile tests}

In order to provide a first insight into the mechanical properties of the blends, specimens of extruded fibers with varying compositions were selected and prepared for tensile testing: PHB, PHB/PCL 90/10, $70 / 30,50 / 50$ from granulate material and additionally PHB/PCL 70/30 from precipitated material to determine the influence of pre-mixing of both components. Mechanical testing was performed with a standard testing machine from the company Zwick (UPM Z010 Zwick GmbH \& Co. KG, Ulm/Germany). The tensile tests were carried out with an overall sample length was $60 \mathrm{~mm}$ and a gauge length of test specimens of $20 \mathrm{~mm}$. The diameter was measured for each specimen separately as it varied from 0.4 to $1.0 \mathrm{~mm}$. A defined preload of $0.1 \mathrm{~N}$ was applied on the specimen prior to the measurement. The crosshead speed was maintained at $10 \mathrm{~mm} / \mathrm{min}$. Tensile properties were calculated as mean value of at least 3 specimens from the obtained stress-strain curves, considering the varying diameter. 


\subsubsection{Scanning Electron Microscopy (SEM)}

Scanning electron microscopy was performed with an environmental scanning electron microscope from Philips (XL30 ESEM-FEG, Philips Deutschland GmbH, Hamburg/Germany) in SEM (highvacuum) mode. To investigate the morphology cross sections of the specimens (compounded PHB/PCL 70/30 from granulate, powder, precipitated material) were imaged. All specimens were submerged into liquid nitrogen and fractured or cut and then sputter-coated with a thin layer of gold before SEM observation.

\subsubsection{Melt spinning}

As a result of the investigation of extruded fibers, the transfer to melt spinning experiments was carried out for the PHB/PCL 70/30 blend only. Melt spinning tests were performed using a self-constructed plunger-piston spinning device:

The spinning device, a bi-component spinning system with plunger and piston, was formerly designed and utilized for core-shell fiber spinning. As described elsewhere, compressed air was filled in as core content instead of the second polymer component to fabricate continuous hollow fibers [7]. About $10 \mathrm{~g}$ of polymer mixture (granulate material, powder and precipitated material, respectively) was filled in the piston whereas the plunger was already positioned. The piston and plunger were connected to the spinning block and the set-up was heated up to $185^{\circ} \mathrm{C}$. The spinneret is available in the dimension of $0.3 / 0.6$ and $0.4 / 0.7 \mathrm{~mm}$ inner/outer diameter. The mass throughput of the spinneret was set to $0.5-$ $1 \mathrm{~cm}^{3} \mathrm{~min}^{-1}$. The resulting spinning pressure was in a range of 30 up to 100 bar. Spun fibers were wound up on a bobbin with an up-take speed varying between 20 up to $100 \mathrm{mmin}^{-1}$. To adjust the inner diameter and the wall thickness of the hollow fibers the up-take speed (draw down ratio) was varied [24, 25]. The higher the applied draw down ratio is set, the smaller the obtained tube diameter. A low dosage of compressed air was used as core component to generate a hollow structure during melt spinning.

\section{Results and discussion}

\subsection{Thermal analysis}

DSC measurements of compounded blend systems were conducted to investigate whether there is miscibility or phase segregation for a particular compo-

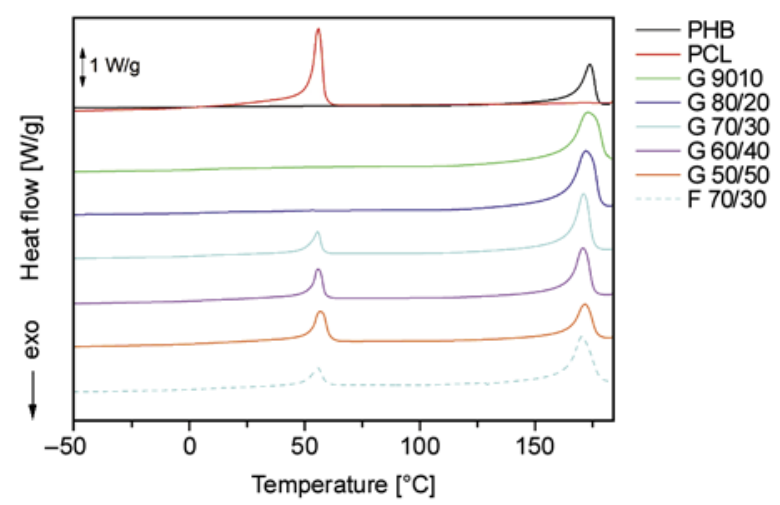

Figure 1. DSC second heating scans of pure PHB and pure PCL compared to PHB/PCL blends

sition, and the influence of PCL on the crystallization behaviour of PHB. The second heating curves, shown in Figure 1, reflected the immiscibility by the occurrence of two glass transitions at temperatures according to that of the components. With increasing PCL content the glass transition of PHB was overlapped by the melting of PCL. In Figure 2 the cooling cycle for the pure components in comparison to several blends is shown. These measurements are in complete accordance with the findings of Lovera et al. [19]: PHB crystallization temperature is shifted from $116^{\circ} \mathrm{C}$ to significantly lower temperatures (varying from $101-104^{\circ} \mathrm{C}$ for $\mathrm{G}$ specimens) when blended with PCL, demonstrating the disturbing effect of PCL on the PHB crystallization. This may be explained by a transfer of heterogeneities from PHB to PCL during blending. The shift to lower $T_{\mathrm{c}}$ is even more distinct $\left(T_{\mathrm{c}}=90^{\circ} \mathrm{C}\right.$ for $\mathrm{F} 70 / 30$ ) for $\mathrm{F} 70 / 30$. This effect can be attributed to the removal of several heterogeneities during the preparation procedure in solution. When the total amount of heterogeneities is decreased while the transfer of these from PHB towards PCL occurs in

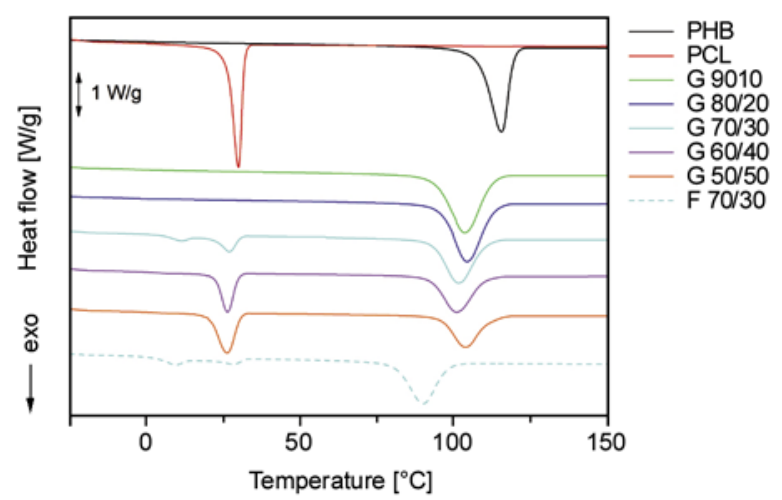

Figure 2. DSC cooling curves of pure PHB compared to $\mathrm{PHB} / \mathrm{PCL}$ blends of varying composition 
the same manner, PHB crystallization happens at significantly lower temperatures.

PCL crystallization is also shifted to lower temperatures for all blended specimens, indicating immiscibility. The G $90 / 10$ as well as the $\mathrm{G} 80 / 20$ blend shows a very small exotherm with $\Delta H_{\mathrm{c}} \leq 0.5 \mathrm{~J} / \mathrm{g}$ for PCL at lower temperatures than the bulk crystallization. In $\mathrm{G} 70 / 30$ as well as in F 70/30 two distinct PCL exotherms occur with lower crystallization temperatures compared to pure PCL. This phenomenon is referred as fractionated crystallization. The small effect of fractionated crystallization was also observed for the composition of $\mathrm{PHB} / \mathrm{PCL}$ $65 / 35$, but is not shown in Figure 2. The first crystallization step with lower supercooling corresponds to the bulk crystallization of PCL. The second one is a typical consequence of fine dispersed regions of the minor phase into isolated droplets within the matrix polymer. Nucleation is not induced at the same temperature for all PCL droplets. The crystallization takes place at different supercoolings due to the varying content and distribution of heterogeneities that are inherently existent and were donated by PHB within the dispersed droplets. The quantity of such crystallization steps varies and is dependent on the number and distribution of nucleating sites in the PCL phase. Notwithstanding that PHB and PCL are immiscible; it is assumed that a homogenously finely dispersed phase showing good interfacial adhesion could lead to improved properties.

For PHB/PCL $60 / 40$ and 50/50 the phenomenon of fractionated crystallization is absent. Presumably in both blend systems both phases, PHB and PCL, coexist without droplet formation in a rather cocontinuous alignment. Figure 2 shows well defined exotherms for both blends confirming the immiscibility of the components. The determined enthalpies verify the amount of both polymers, respectively.

\subsection{Mechanical performance}

Taking the results of the DSC measurement into account, samples of selected compositions have been chosen for the tensile tests. Extruded fibers have been evaluated: pure PHB, PHB/PCL 90/10, $70 / 30$ and $50 / 50$ from granulate mixture $(G)$ as well as $70 / 30$ from precipitated material (F) have been tested. The given values by Perstorp for the mechanical properties of PCL capa ${ }^{\circledR} 6800$ have been veri-

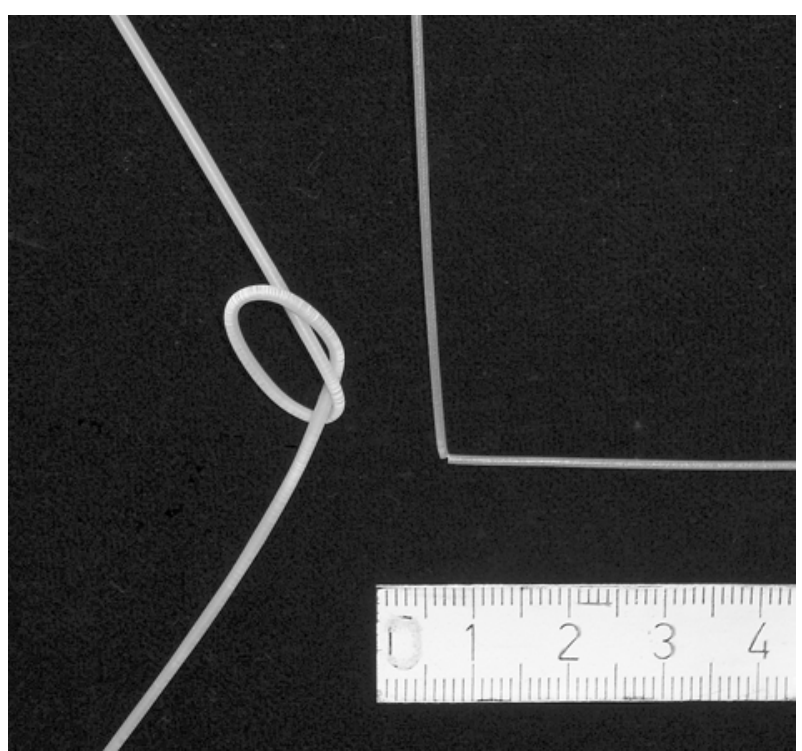

Figure 3. Image of extruded 'worm-like' structures to visualize the flexibility: left) PHB/PCL 70/30 (flakes) exhibits bendable properties, right) pure $\mathrm{PHB}$ shows brittle-fracture

fied. The aim of these measurements was to demonstrate and to prove the improved flexure of different blends compared to pure PHB and to chose a reasonable composition.

A significant difference occurs in bending stiffness comparing $\mathrm{PHB} / \mathrm{PCL} 70 / 30$ with pure $\mathrm{PHB}$, as demonstrated in Figure 3. Thinking of the desired application the tubular structure will neither be exposed to high tensile or compressive forces nor to extreme torsion or bending as demonstrated with this image. However, certain flexibility or rather pliability is required to prevent tubular structure from kinking or catastrophically failure. A bending test would be the optimal testing method to verify the improved characteristic. However, the available sample geometry (rod like structures) and the available testing equipment does not allow for appropriate standardized bending tests. Thus, tensile tests were carried out to demonstrate the tendency of an improved ductility, even though the bending stiffness was not measured directly.

At first view it seems that there is no significant improvement of ductility present when comparing PHB to the blends. The values for elongation at yield stress, shown in Figure 4 and listed in Table 1, are in a range of $2-4 \%$ for all measured specimens. However, to evidence an increased elasticity the elastic modulus (Young's modulus, $E$ ) shows a trend: $E$ is clearly decreased with increasing PCL content 
Table 1. Mechanical properties of pure components compared to extruded blend fibers

\begin{tabular}{|l|c|c|c|c|c|}
\hline & $\begin{array}{c}\text { Young's modulus } \\
{[\mathbf{G P a}]}\end{array}$ & $\begin{array}{c}\text { Yield stress } \\
{[\mathbf{M P a}]}\end{array}$ & $\begin{array}{c}\text { Elongation at yield stress } \\
{[\%]}\end{array}$ & $\begin{array}{c}\text { Stress at break } \\
{[\mathbf{M P a}]}\end{array}$ & $\begin{array}{c}\text { Elongation at break } \\
{[\%]}\end{array}$ \\
\hline G PHB $(n=8)$ & $1.5 \pm 0.06$ & $26.5 \pm 1.4$ & $2.9 \pm 0.3$ & $25.9 \pm 1.5$ & $3.1 \pm 0.5$ \\
\hline G 90/10 $(n=5)$ & $1.5 \pm 0.17$ & $25.5 \pm 1.8$ & $2.6 \pm 0.2$ & $21.8 \pm 1.3$ & $11.3 \pm 4.9$ \\
\hline G 70/30 $(n=5)$ & $1.3 \pm 0.14$ & $24.4 \pm 1.4$ & $2.9 \pm 0.3$ & $7.0 \pm 8.4$ & $41.2 \pm 22.5$ \\
\hline G 50/50 $(n=3)$ & $1.1 \pm 0.19$ & $22.8 \pm 0.4$ & $3.6 \pm 0.7$ & $27.0 \pm 3.6$ & $630.9 \pm 271.8$ \\
\hline F 70/30 $(n=7)$ & $1.3 \pm 0.15$ & $25.4 \pm 1.7$ & $3.1 \pm 0.6$ & - & - \\
\hline PCL (Perstorp) & 0.5 & 16 & - & 54 & 920 \\
\hline
\end{tabular}
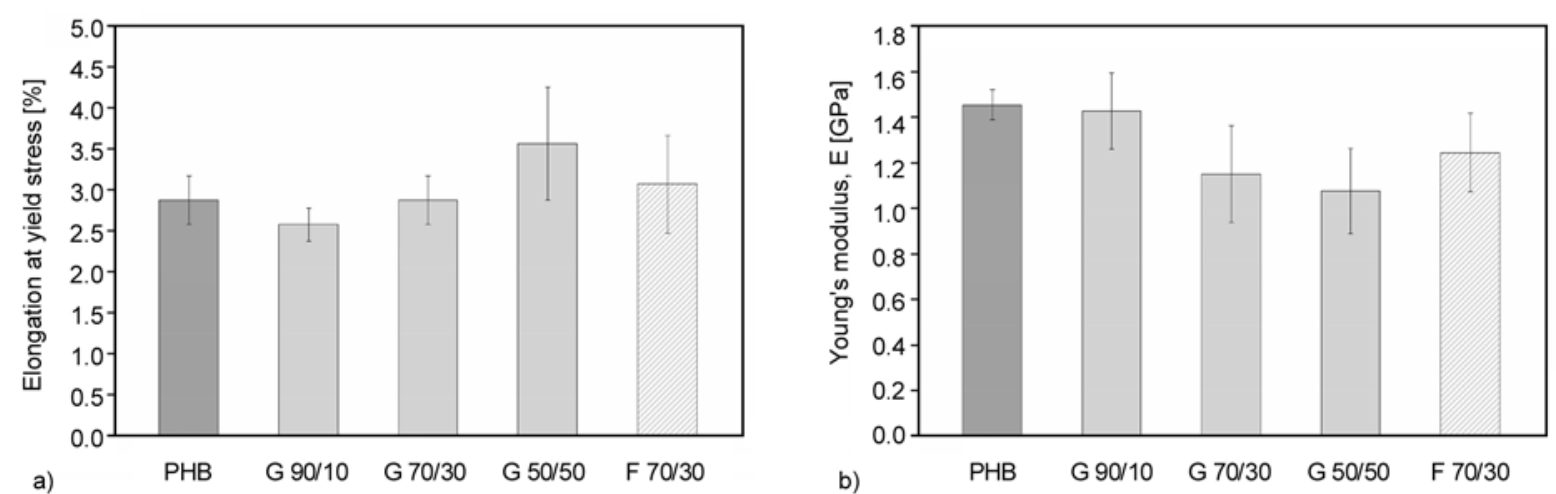

Figure 4. Mechanical properties determined from tensile tests: elongation at yield stress (a) and Young's modulus (b) (F = flakes from precipitated material; ${ }^{*}$ given by Perstorp for capa ${ }^{\circledR} 8600$ )

as diagrammed with Figure 4. In order to understand and evaluate the determined values correctly some insight into the fracture behaviour needs to be given. It is known that PHB shows brittle-fracture, which is exactly what was observed within the test: maximal elongation $(2.9 \%)$ is equal to the elongation at break (3.1\%). The addition of $10 \mathrm{wt} \% \mathrm{PCL}$ (G 90/10) already yields into an increased value for elongation at break. While elongation at yield stress is in the same range $(2.6 \%)$ the elongation at break increased to $11.3 \%$. Nevertheless, these specimens also show brittle-fracture.

However, the blend systems $\mathrm{G} 70 / 30$ as well as G 50/50 show elongation with necking. Even though elongation at yield stress remains only about $3-4 \%$, no brittle-fracture occurs in these cases, instead samples start to neck. For these specimens elongation at break is in the range from 10 up to $800 \%$, respectively. Admittedly, a great variance occurs for the values of elongation at break for these blends. This might be explained by inhomogeneities in blend compositions and morphology that strongly affect the necking behaviour.

Considering the desired application, it is important to mention here that elongation at break is not required to be extremely high or even as high as for pure PCL (920\%). In this specific case it is necessary to prove that brittle-fracture can be prevented and a recognizable pliability can be obtained by blending PHB with PCL. The data for elongation at yield stress and the Young's modulus are in the similar range for $\mathrm{G} 70 / 30$ compared to $\mathrm{G} 50 / 50$. However, the failure behaviour differs since G 50/50 exhibits a significantly higher elongation at break $(630.9 \%)$. Consequently, the addition of only $30 \mathrm{wt} \%$ of PCL is reasonable and adequate to adjust the desired characteristic in terms of avoiding brittle fracture and obtaining a bendable material. The aim of the study was the improvement of PHB properties by using a minimum amount of a second polymer.

Moreover, it needs to be mentioned that whitish horizontal stripes at macroscopic scale have been detected prior failure as it can be seen in Figure 3, indicating a certain material damage. The stripes occur at the knot sites for the regions of maximum of deformation; they are irreversible and are related to local crazing. Therefore, specimens can not be considered flexible but bendable.

To address the question of mixture homogeneity, which is essential for melt spinning, extruded fibers from precipitated material $\mathrm{F}$ 70/30 were compared to extruded fibers made from granulate material G 70/30. From the tensile test data no significant difference can be observed, except for elongation at break. No reliable data for elongation at break for 


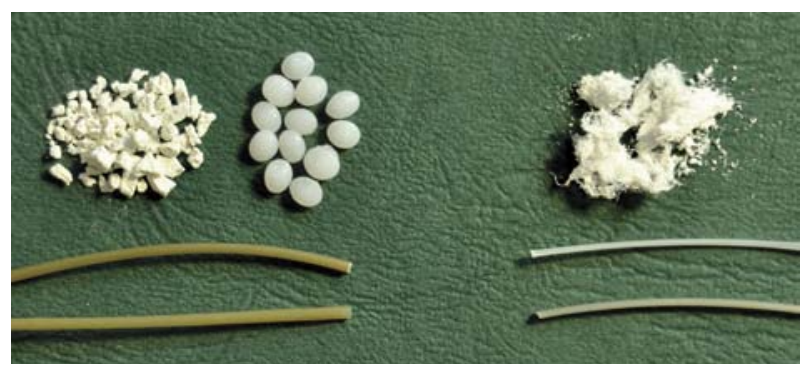

Figure 5. Compounded specimens from G 70/30 left (brownish) compared to $\mathrm{F} 70 / 30$ (whitish) right

F 70/30 was measured because specimens showed failure out of gauge length in nearly all cases. Breaking behaviour can be considered similar to $\mathrm{G} 70 / 30$ : G 70/30 as well as F 70/30 showed elongation with necking instead of catastrophically failure. Both mixtures showed equal behaviour in terms of its bending properties.

As it is visualized in Figure 5, it is remarkable that precipitated blend material results into whitish almost transparent appearing samples, whereas compounded granules look brownish after compounding. Aesthetical optical appearance is definitively an issue in medical applications. Even more important is the well pre-mixed state of both components which is essential for further spinning experiments (see 2.3). Mixing of PHB and PCL in solution and subsequent precipitation leads to a well mixed state, to purification of the material and to the removal of low molecular constituents, which show higher sensibility to thermooxidative degradation. Thus, purified $\mathrm{F} 70 / 30$ specimen exhibits a rather white appearance after compounding compared to the G 70/30 sample that contains more impurities and low molecular constituents.

\subsection{Melt spinning}

Finally, spinning experiments were conducted using the plunger-piston spinning device due to two advantages: first, the chance to use a minimum of material and the simplified handling of the spinning device compared to extrusion spinning and second the opportunity to spin at lower temperatures than $190^{\circ} \mathrm{C}$ to avoid thermooxidative degradation. Spinning of hollow fibers was successfully conducted at temperatures in the range of $183-185^{\circ} \mathrm{C}$. As the premixing of blends is essential for spinning of homogenous fibers, different mixing states were tested: granulate material, powder and precipitated material. The spinning procedure for granulates as well

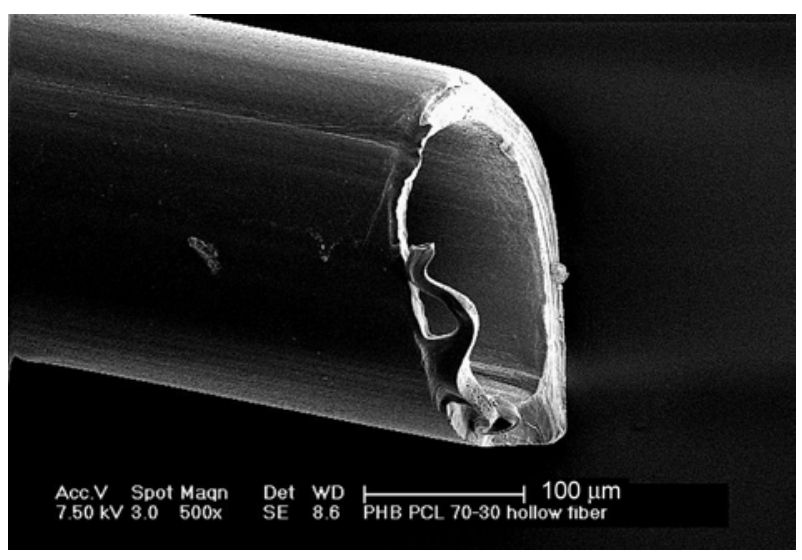

Figure 6. SEM image of hollow melt spun fiber from PHB/PCL 70/30

as for powder mixture was instable and seemed to yield into segregation of both components during spinning. PCL melts at approximately $60^{\circ} \mathrm{C}$, whereas PHB melting starts at $175^{\circ} \mathrm{C}$. Thus, PCL melt already moves towards the nozzle by gravity yielding into phase segregation within the plunger. $\mathrm{PHB} / \mathrm{PCL}$ 70/30 from precipitated material (F 70/30) did not show phase separation phenomena and thus melt spinning could be carried out successfully. Melt spinning of compacted flakes resulted in flexible hollow fibers of adequate optical and mechanical appearance. A SEM image of a hollow fibre is shown in Figure 6. Dimensionally stable and homogenous hollow fibers with smooth inner and outer tube surfaces have been fabricated. The rather flexible properties might be visualized by the soft cut leading to plastic deformation, which would not be visible for a brittle-fracture.

Through variation of the up-take speed and by dosage of the air pressure the diameter and the wall thickness of the hollow fibers was adjusted. The maximal diameter and wall thickness were limited by the geometry of the nozzle. By increasing the up-take speed of the bobbin (at constant mass throughput) a higher draw down ratio was set. The result was a reduced fiber diameter and a decreased wall thickness. The inner diameters of the fabricated tubes were in a range from 50 up to $600 \mu \mathrm{m}$.

\subsection{Morphology}

Figure 7 shows cryo-fractured cross sections of specimens of PHB/PCL 70/30 (G, P, F) that have been made by means of extrusion. The two phase system can be clearly seen for a) and b). Small homogenously dispersed droplets of PCL within the 


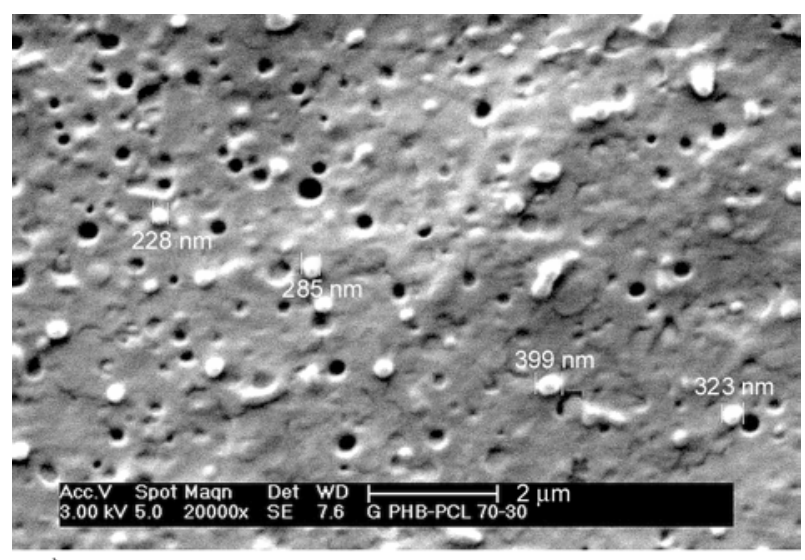

a)

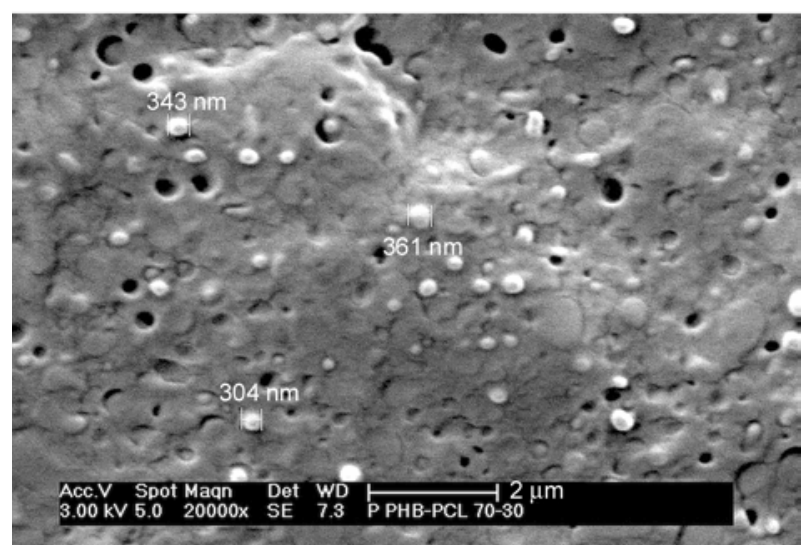

b)

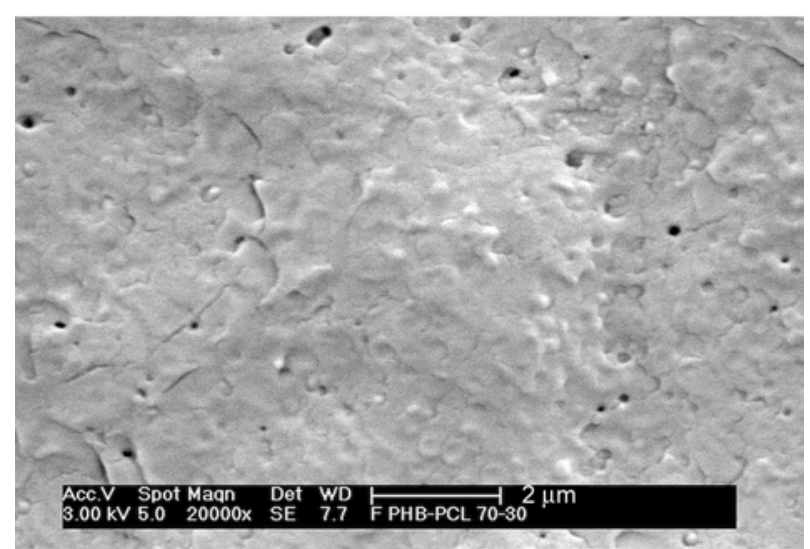

c)

Figure 7. SEM images of extruded PHB/PCL 70/30 specimens: a) mixed granulates, b) powder mixture, c) precipitated material

PHB matrix are visible. Droplet size is in the range $200-400 \mathrm{~nm}$. Some of them seem to be off place due to cryo-treatment in liquid nitrogen. PCL droplets might fall off at the surface of fracture since it is brittle at temperatures below $-60^{\circ} \mathrm{C}\left(T_{\mathrm{g}}\right)$. For the precipitated material such droplet structure is hardly visible. This procedure enables mixing on lowest possible molecular scale. Mixing in solution is therefore regarded to lead to the best available mixture for PHB/PCL 70/30 for spinning experiments.

\section{Conclusions}

Using a PHB/PCL 70/30 blend system dimensionally stable hollow biocompatible and biodegradable fibers have been produced by means of melt spinning. It was shown that a blend from precipitated material was well processable, whereas granulate material or powder mixtures were not spinnable due to phase separation phenomena. Contrary to other findings, tensile measurements showed evidence for significantly improved elastic properties for this immiscible blend in terms of bending stiffness. Brittle-fracture and catastrophical failure, as it is known for pure PHB, did not occur for the particular blend. Crazing deformation has been detected at the regions of maximum bending. However, the further application of the hollow fibers is not associated with maximum bending, high tension or compression stress. Thus, the achieved improvement of mechanical properties is satisfying.

For a composition of PHB/PCL 70/30 a microphase separation (fractionated crystallization) has been detected yielding into a fine homogenous dispersion of PCL droplets within in the matrix phase. However, the stated assumption that a fine dispersion of PCL droplets within the PHB matrix will lead to improved properties was not proven. A composition of 50/50, for which no droplet dispersion is visible, shows no significant difference in terms of flexibility compared to 70/30. Supposedly, the reason for better mechanical performance is a good interfacial adhesion between the different phases independent of their dispersion. A minimum of $30 \mathrm{wt} \%$ PCL is sufficient to adjust the mechanical properties towards the desired application in this case. The different contents of PCL may be useful to tailor the degradation rate. Providing adequate biological, physical and mechanical properties and a relevant biomolecular surface functionalization hollow fibers made from this specific blend are considered to be advanced structures for tissue engineering purposes, for instance as nerve guidance conduits. A certain porosity for permeation of nutrients and metabolites, one of the most important but challenging requirements for this particular application, has not been adjusted yet. The genera- 
tion of a defined porous hollow fibers based on PHB will be subject of further investigations.

\section{Acknowledgements}

The authors would like to thank K. Scheibe, Dr. H. Scheibner and Dr. K. Schneider for carrying out the tensile measurements and the lively discussions.

Financial support from the Leibniz Institute for Polymer Research Dresden e.V. (Departments: Mechanics and Structure, and Biofunctional Polymer Materials) and the German Research Foundation (Ni 709/3-1) is gratefully acknowledged.

\section{References}

[1] Chen G-Q., Wu Q.: The application of polyhydroxyalkanoates as tissue engineering materials. Biomaterials, 26, 6565-6578 (2005).

DOI: $10.1016 /$ j.biomaterials.2005.04.036

[2] Freier T.: Biopolyesters in tissue engineering applications. Polymers for Regenerative Medicine, 203, 1-61 (2006).

DOI: $10.1007 / 12073$

[3] Rentsch C., Rentsch B., Breier A., Hofmann A., Manthey S., Scharnweber D., Biewener A., Zwipp H.: Evaluation of the osteogenic potential and vascularization of 3D poly(3)hydroxybutyrate scaffolds subcutaneously implanted in nude rats. Journal of Biomedical Materials Research Part A, 92, 185-195 (2010).

DOI: $10.1002 /$ jbm.a.32314

[4] Schmack G., Jehnichen D., Vogel R., Tändler B.: Biodegradable fibers of poly(3-hydroxybutyrate) produced by high-speed melt spinning and spin drawing. Journal of Polymer Science Part B: Polymer Physics, 38, 2841-2850 (2000).

DOI: $10.1002 / 1099-0488(20001101) 38: 21<2841:: A I D-$ POLB130>3.0.CO;2-\#

[5] Vogel R., Tändler B., Häussler L., Jehnichen D., Brünig H.: Melt spinning of poly(3-hydroxybutyrate) fibers for tissue engineering using $\alpha$-cyclodextrin/ polymer inclusion complexes as the nucleation agent. Macromolecular Bioscience, 6, 730-736 (2006).

DOI: $10.1002 / \mathrm{mabi} .200600116$

[6] Kil'deeva N. R., Vikhoreva G. A., Gal'braikh L. S., Mironov A. V., Bonartseva G. A., Perminov P. A., Romashova A. N.: Preparation of biodegradable porous films for use as wound coverings. Applied Biochemistry and Microbiology, 42, 631-635 (2006).

DOI: $10.1134 / \mathrm{S} 0003683806060160$

[7] Hinüber C., Häussler L., Vogel R., Brünig H., Werner C.: Hollow poly(3-hydroxybutyrate) fibers produced by melt spinning. Macromolecular Materials and Engineering, 295, 585-594 (2010).

DOI: $10.1002 /$ mame.201000023
[8] Battiston B., Geuna S., Ferrero M., Tos P.: Nerve repair by means of tubulization: Literature review and personal clinical experience comparing biological and synthetic conduits for sensory nerve repair. Microsurgery, 25, 258-267 (2005).

DOI: $10.1002 /$ micr.20127

[9] Ruiter G. C. W., Malessy M. J. A., Yaszemski M. J., Windebank A. J., Spinner R. J.: Designing ideal conduits for peripheral nerve repair. Neurosurgical Focus, 26, E5/1-E5/9 (2009).

DOI: 10.3171/FOC.2009.26.2.E5

[10] Yao L., de Ruiter G. C. W., Wang H. A., Knight A. M., Spinner R. J., Yaszemski M. J., Windebank A. J., Pandit A.: Controlling dispersion of axonal regeneration using a multichannel collagen nerve conduit. Biomaterials, 31, 5789-5797 (2010).

DOI: $10.1016 /$ j.biomaterials.2010.03.081

[11] Novikova L. N., Pettersson J., Brohlin M., Wiberg M., Novikov L. N.: Biodegradable poly- $\beta$-hydroxybutyrate scaffold seeded with Schwann cells to promote spinal cord repair. Biomaterials, 29, 1198-1206 (2008). DOI: 10.1016/j.biomaterials.2007.11.033

[12] Terenghi G.: Peripheral nerve regeneration and neurotrophic factors. Journal of Anatomy, 194, 1-14 (1999). DOI: $10.1046 / \mathrm{j} .1469-7580.1999 .19410001 . \mathrm{X}$

[13] Jiang X., Lim S. H., Mao H-Q., Chew S. Y.: Current applications and future perspectives of artificial nerve conduits. Experimental Neurology, 223, 86-101 (2010). DOI: 10.1016/j.expneurol.2009.09.009

[14] Qian Z. Y., He Y., Zou Y. B., Li S., Liu X. B.: Structure and property study of degradable polyesteramide fibres: Processing and alkaline degradation behaviour. Polymer Degradation and Stability, 83, 127-132 (2004). DOI: 10.1016/S0141-3910(03)00233-7

[15] Belkas J. S., Munro C. A., Shoichet M. S., Johnston M., Midha R.: Long-term in vivo biomechanical properties and biocompatibility of poly(2-hydroxyethyl methacrylate-co-methyl methacrylate) nerve conduits. Biomaterials, 26, 1741-1749 (2005). DOI: 10.1016/j.biomaterials.2004.05.031

[16] Hazari A., Johansson-Rudén G., Junemo-Bostrom K., Ljungberg C., Terenghi G., Green C., Wiberg M.: A new resorbable wrap-around implant as an alternative nerve repair technique. The Journal of Hand Surgery, European Volume, 24, 291-295 (1999). DOI: 10.1054/JHSB.1998.0001

[17] Mohanna P-N., Young R. C., Wiberg M., Terenghi G.: A composite poly-hydroxybutyrate-glial growth factor conduit for long nerve gap repairs. Journal of Anatomy, 203, 553-565 (2003). DOI: $10.1046 / \mathrm{j} .1469-7580.2003 .00243 . \mathrm{x}$

[18] Avella M., Martuscelli E., Raimo M.: Review properties of blends and composites based on poly(3-hydroxy) butyrate (PHB) and poly(3-hydroxybutyrate-hydroxyvalerate) (PHBV) copolymers. Journal of Materials Science, 35, 523-545 (2000). DOI: $10.1023 / \mathrm{A}: 1004740522751$ 
[19] Lovera D., Márquez L., Balsamo V., Taddei A., Castelli C., Müller A. J.: Crystallization, morphology, and enzymatic degradation of polyhydroxybutyrate/ polycaprolactone (PHB/PCL) blends. Macromolecular Chemistry and Physics, 208, 924-937 (2007).

DOI: $10.1002 /$ macp.200700011

[20] Jenkins M. J., Cao Y., Howell L., Leeke G. A.: Miscibility in blends of poly(3-hydroxybutyrate-co-3-hydroxyvalerate) and poly( $\varepsilon$-caprolactone) induced by melt blending in the presence of supercritical $\mathrm{CO}_{2}$. Polymer, 48, 6304-6310 (2007).

DOI: $10.1016 /$ j.polymer.2007.08.033

[21] Qiu Z. B., Fujinami S., Komura M., Nakajima K., Ikehara T., Nishi T.: Structure and properties of biodegradable polymer-based blends. Macromolecular Symposia, 216, 255-263 (2004).

DOI: $10.1002 /$ masy.200451224
[22] Chee M. J. K., Ismail J., Kummerlöwe C., Kammer H. W.: Study on miscibility of PEO and PCL in blends with PHB by solution viscometry. Polymer, 43, 1235-1239 (2002).

DOI: $10.1016 / \mathrm{S} 0032-3861(01) 00725-\mathrm{X}$

[23] Gassner F., Owen A. J.: Physical properties of poly( $\beta$ hydroxybutyrate)-poly( $\varepsilon$-caprolactone) blends. Polymer, 35, 2233-2236 (1994).

DOI: 10.1016/0032-3861(94)90258-5

[24] Beyreuther R., Brünig H.: Dynamics of fibre formation and processing. Springer, Berlin (2006).

[25] Ziabicki A.: Fundamentals of fiber formation: The science of fibre spinning and drawing. Wiley, New York (1976). 